


\section{ABSORPTION SPECTRUM OF THE NO MOLECULE}

\section{SURVEY OF EXCITED STATES AND THEIR INTERACTIONS}

K. DRESSLER

Department of Astrophysical Sciences, Princeton University and

\section{E. MIESCHER}

Institute of Physics, University of Basel, Basel, Switzerland

\section{ABSTRACT}

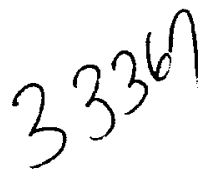

New analyses of absorption bands of NO, based on high resolution spectrograms of three isotopic NO molecules in the wavelength region 1550 - $1400 \AA$, are reported. The rotational and vibrational structures as well as the intensities of the observed bands are largely determined by the numerous interactions found between Rydberg-type and non-Rydberg-type energy levels of identical symmetry. Several new Rydberg states of symmetries ${ }^{2} \Sigma^{+},{ }^{2} \Pi,{ }^{2} \Delta$ are established and ordered into a consistent scheme. A value of $I\left(N O, X^{2} \Pi_{\frac{1}{2}}\right)=9.266 \mathrm{eV}$ is obtained from a Rydberg extrapolation. The non-Rydberg states resulting from configurations $\sigma \pi^{2}$ and $\pi^{3} \pi^{2}$ have all been identified except for $\mathrm{a}^{2} \Phi$ state. In particular, some sharp as well as some recognizable diffuse levels belonging to the state $\left(\sigma \pi^{2}\right)$ $I^{2} \Sigma^{+}$have been found in spite of the presence at those energies of the repulsive state $(\bar{\sigma} 2 \mathrm{p}) \mathrm{A}^{\prime 2} \Sigma^{+}$arising from ground state atoms $\mathrm{N}+\mathrm{O}$. The numerical results are presented in Tables $1-3$.

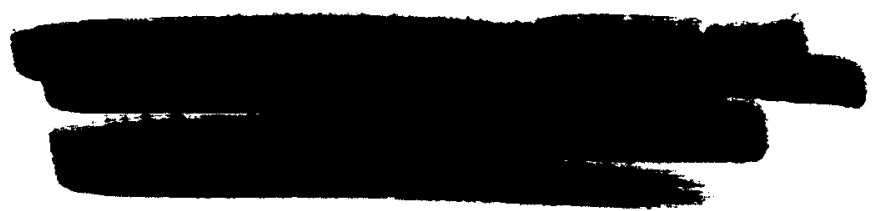




\section{INTRODUCTION}

The detailed study of the absorption spectrum of nitric oxide was initiated a few years ago when plates taken with vacuum spectrographs of high resolving power became available (Lagerqvist and Miescher 1958, 1962; Huber and Miescher 1963; Lofthus and Miescher 1964). It has since been demonstrated that this molecule is particularly well suited for a detailed investigation of the excited states of a molecular system up to the limit of ionization. Owing to the comparatively low ionization potential of NO (9.27 eV) the interesting part of the spectrum of this molecule, in contrast to, e.g., $\mathrm{N}_{2}$ or $\mathrm{O}_{2}$, falls into the region of longer wavelengths $(\lambda \geq 1200 \AA)$ which has become accessible in recent years with the most powerful instruments. Throughout the entire Schumann region down to $1200 \AA$ the absorption spectrum of NO is full of sharp bands which can be resolved today into fine structure due to rotation, electron spin and $\Lambda$-doubling. The structure of this spectrum is extremely complex and the ordering of the observed bands into electronic or vibrational systems is by no means obvious; neither Rydberg series nor band convergences can immediately be recognized; however, the detailed analysis has now resulted in a progressive understanding of the observations.

As has been stated repeatedly, the system of excited states of NO is characterized by a clear division into two separate classes, viz., Rydberg- (R-) and non-Rydberg-(NR-) states (see Fig. la). In the R-

states... $(\sigma 2 p)^{2}(\pi 2 p)^{4} \lambda n \ell, n \geq 3$, one single electron is excited into a Rydberg-type orbital and its energy is of no consequence with respect 
to the properties of the ${ }^{1} \Sigma^{+}$molecular core. The $R$ - states form the $\ell-$ complexes shown in Fig. la. The NR- states result from the unfilledshell configurations of the $2 \mathrm{p}$ electrons and they are characterized by considerably looser binding in the molecule:

$$
\begin{aligned}
& \ldots(\sigma 2 \mathrm{p})^{2}(\pi 2 \mathrm{p})^{4} \bar{\sigma} 2 \mathrm{p}: \mathrm{A}^{\prime}{ }^{2} \Sigma^{+}, \\
& \ldots \sigma 2 \mathrm{p}(\pi 2 \mathrm{p})^{4} \overline{(\pi 2 \mathrm{p})^{2}:} \mathrm{B}^{\prime 2} \Delta, \mathrm{G}^{2} \Sigma^{-}, \mathrm{I}^{2} \Sigma^{+},\left(\mathrm{b}^{4} \Sigma^{-}\right), \\
& \ldots(\sigma 2 \mathrm{p})^{2}(\pi 2 \mathrm{p})^{3}(\bar{\pi} 2 \mathrm{p})^{2}: \mathrm{B}^{2} \Pi, \mathrm{L}^{2} \Pi, \mathrm{P}^{2} \Pi, \mathrm{L}^{\prime 2} \Phi,\left(\mathrm{a}{ }^{4} \Pi\right) .
\end{aligned}
$$

Only doublet states will be further discussed in this paper.

Fig. lb shows schematic potential curves of a $R$ - and a NR - state and of the ground state. It is evident that the zero order R-and NRcurves cross each other; therefore, strong interactions between $\mathrm{R}$ - and NR - states of the same symmetry can occur. Since the magnitudes of the rotational constants in $\mathbf{R}$ - states are much larger than those in $\mathrm{NR}$ - states, the total energies $W=T+B J(J+1)$ of pairs of $R-$ and $N R-$ states are often found to cross each other, as illustrated schematically in Fig. 2 . The observed rotational term sequences are the perturbed $W^{\prime}=T \pm \epsilon+F(J)$, where $F(J)$ is described by $B$ - values which are no longer constant but functions of $\mathrm{J}$ defined as

$$
B(J)=\frac{\Delta_{1} F}{2(J+1)} \text { or } B(J)=\frac{\Delta_{2} F}{4 J+2} \text {. }
$$

The magnitude of the interaction energy $H$ varies greatly from case to case. These perturbations are widely responsible for the structure of the observed spectrum and its apparent complexity, and they are responsible also for the evident nonsimilarity between the band structures 
observed in the spectra of different isotopic molecules $\mathrm{N}^{14} \mathrm{O}^{16}, \mathrm{~N}^{15} \mathrm{O}^{16}$, $\mathrm{N}^{14} \mathrm{O}^{18}$. Since in $\mathrm{R}$ - as well as in NR- states the equilibrium internuclear distance differs considerably from that in the ground state, the absorption spectrum consists of long vibrational progressions; i.e., the majority of the observed energy levels contain a large amount of vibrational energy which varies depending on the mass of the isotope. For this reason the relationship between interacting vibrational levels can be quite different from isotope to isotope.

On the other hand the determination of the symmetry type $(\Sigma, \Pi, \Delta)$ of $\mathrm{R}$ - states is strongly supported by observations of just these interactions between R- and NR- levels. It is difficult to distinguish between unperturbed $R$ - levels since they all have a vanishingly small spin splitting and identical values of $B$ and $\omega$, whereas the NR-states show considerable variation in their properties. Strong interactions occur only between Rand NR- levels for states of identical symmetry (homogeneous perturbation). $\Sigma \sim \Sigma$ interactions do not involve the levels of the NR - state $G^{2} \Sigma^{-}$ (cf., Lofthus and Miescher 1964), since there exist no R-states of ${ }^{2} \Sigma^{-}$ symmetry. ${ }^{2} \Sigma^{+} R$ - states show many predissociations caused by the repulsive $\mathrm{A}^{\prime 2} \Sigma^{+}$state arising from ground state atoms, whereas perturbed band structure due to interaction with the NR-state $I^{2} \Sigma^{+}$has been found in only one $\mathrm{R}$ - level. ${ }^{2} \Pi \sim^{2} \Pi$ interactions are much more complicated because they involve the levels of three ${ }^{2} \Pi$ NR - states, each having a large spin doublet separation, namely the high vibrational levels $v>21$ of $B^{2} \Pi$ and the higher lying states $L^{2} \Pi$ and $P^{2} \Pi$. At these high 
energies it is difficult to distinguish between these three ${ }^{2} \Pi$ NR - states which to a certain extent mix with each other. Much simpler and strikingly analogous to the $R \sim N R$ interactions between $C^{2} \Pi(v \leq 4)$ and $\mathrm{B}^{2} \Pi(v \leq 19)$ studied by Lagerqvist and Miescher (1958) are the mutual interactions in the ${ }^{2} \Delta$ states. As only one NR-state, $B^{\prime 2} \Delta$, exists, the ${ }^{2} \Delta R$ - levels are plainly discerned from the observed perturbations in the $B^{\prime 2} \Delta$ NR- levels.

Compared with the discussed homogeneous interations $(\Sigma \sim \Sigma, \Pi \sim \Pi$, $\Delta \sim \Delta)$ heterogeneous perturbations $(\Sigma \sim \Pi, \Pi \sim \Delta)$ are small; nevertheless they are perceptible, be it as $\Lambda$-doubling in $R \sim R$ interactions or as anomalous doublet splitting in $\mathbf{R} \sim \mathrm{NR}$ interactions or as predissociation of ${ }^{2} \Pi$ levels by the $A^{\prime 2} \Sigma^{+}$continuum.

In the following three sections the results obtained for ${ }^{2} \Sigma^{+},{ }^{2} \Pi,{ }^{2} \Delta$ levels of $\mathbf{R}$ - as well as of NR-states will be discussed successively. Three tables (Tables $1-3$ ) contain the numerical data. Constants for lower lying levels not included in these tables have been given by Barrow and Miescher (1957) and in the papers quoted at the beginning of this section.

A short description of the experimental setup (10-meter vacuum spectrograph at the National Research Council of Canada, liquid oxygen cooled absorption cell, Lyman continuum) has been given by Lofthus and Miescher (1964).

$$
\text { II. }{ }^{2} \Sigma^{+} \text {STATES }
$$

Two ${ }^{2} \Sigma^{+}$NR - states have been derived from electron configurations 
in Section I. One of these is the repulsive state $A^{\prime 2} \Sigma^{+}$(see Fig. 3), whereas the other one, ${ }^{2} \Sigma^{+}$, would be expected to have a stable minimum at an energy somewhat above that of $\mathrm{B}^{\prime 2} \Delta$. However, at these energies these two ${ }^{2} \Sigma^{+}$NR - states presumably mix appreciably with each other. Experimental evidence for the existence of discrete sharp as well as diffuse ${ }^{2} \Sigma^{+}$ NR - energy levels is presented for the first time here (see Table 1) and demonstrates that the degree of mixing varies considerably from one vibrational level of $\mathrm{I}^{2} \Sigma^{+}$to another and even from one isotope to another at the same vibrational quantum number $v$. The vibrational overlap (FranckCondon factors) between $A^{\prime}$ and I must go through considerable variations in the observed energy range; in particular it must assume very small values at some energies, allowing some levels of $\mathrm{I}^{2} \Sigma^{+}$to be almost free from broadening.

The information now available on the new $I^{2} \Sigma^{+}$NR-state is much more fragmentary than that on the related $G^{2} \Sigma^{-}$and $B^{\prime 2} \Delta$ states. A total of seven observed bands, counting all three isotopes, can be ordered into a vibrational progression as shown in Table 4. Quantum numbers $v$ and system origin $\mathrm{T}_{0}$ are tentatively assigned from the observed vibrational isotope shifts assuming that the band positions are not much perturbed and that the anharmonicity of the I state is similar to that of G and $B^{\prime}\left(\omega x \approx 15 \mathrm{~cm}^{-1}\right)$. Rotational analyses have been carried out for the two sharp bands at $69993 \mathrm{~cm}^{-1}\left(\mathrm{~N}^{14} \mathrm{O}\right)$ and at $68831 \mathrm{~cm}^{-1}\left(\mathrm{~N}^{15} \mathrm{O}\right)$. All other five bands are diffuse. B-values have been estimated for those bands from the measurements of the distance between the two heads 
formed by the $R_{21}$ - branch and the combined $R_{11}+Q_{21}$ - branch, according to the relation for unperturbed bands of type ${ }^{2} \Sigma(b)-{ }^{2} \Pi(a)$ :

$$
\nu\left(\mathrm{R}_{21} \text { - head }\right)-\nu\left(\mathrm{Q}_{21} \text { - head }\right)=2 \mathrm{~B}^{\prime} \mathrm{B}^{\prime \prime} /\left(\mathrm{B}^{\prime}-\mathrm{B}^{\prime \prime}\right)
$$

They all fall within the range 1.30 to $1.40 \mathrm{~cm}^{-1}$ in close agreement with the more accurate $\bar{B}$ - value obtained from the detailed analysis of the sharp band of $\mathrm{N}^{14} \mathrm{O}$ at $69993 \mathrm{~cm}^{-1}$. The band of $\mathrm{N}^{15} \mathrm{O}$ at $68831 \mathrm{~cm}^{-1}$, according to the definition adopted here, actually is a $R$ - band on account of the high value of $B$ it shows at the lowest rotational quantum numbers $\mathrm{J}$ (cf., Tables 1 and 4). Its upper state is mostly the $R$ - state $(\sigma 5 p) R^{2} \Sigma^{+}$ at the lowest $\mathrm{J}$ - value, but with increasing rotation the $B$ - value tends towards the constant value of $1.3 \mathrm{~cm}^{-1}$ which falls well in line with all other observed B-values of the $I^{2} \Sigma^{+} N R$ - levels. This $R \sim N R$ interaction between two ${ }^{2} \Sigma^{+}$states constitutes the only known example for states of this symmetry in NO where a sharp rotational structure is homogeneously perturbed. The interaction energy $\mathrm{H}$ is estimated to be $6 \mathrm{~cm}^{-1}$. Further $R \sim N R$ interactions between ${ }^{2} \Sigma^{+}$states are observed in the form of diffuseness in several ${ }^{2} \Sigma^{+} R$ - levels, owing to strong predissociation by the repulsive state $\mathrm{A}^{\prime 2} \Sigma^{+}$. However, it appears likely that the strong predissociation of $\mathbf{R}$ - levels observed as diffuseness in absorp$\underline{\text { tion }}$ constitutes a case of accidental predissociation, involving three states of identical symmetry; i.e., the predissociation of those ${ }^{2} \Sigma^{+}$R-levels occurs probably indirectly through mixing with vibrational levels of $I^{2} \Sigma^{+}$ which in turn are strongly predissociated by the $\mathrm{A}^{\prime 2} \Sigma^{+}$continuum. The 
i experimental evidence is as follows. The sharp rotational structure of the $R$ - level $Y^{2} \Sigma^{+}(v=0)$ in $N^{14} O$ is observed to break off above $N=5$. This is almost certainly to be interpreted as onset of diffuseness due to mixing with the level $I(v=7)$ which is very diffuse and is observed at $90 \mathrm{~cm}^{-1}$ above the level $\mathrm{Y}(\mathrm{v}=0)$ at $\mathrm{N}=0$. With increasing rotation the levels of $I(v=7)$ must approach those of $Y(v=0)$ owing to the smaller $B-v a l u e$ in the NR-state I, leading to the observed breaking-off in $\mathrm{Y}$ above $\mathrm{N}=5$. In addition to this predissociation of $Y(v=0)$ through mixing with $I(v=7)$ it probably is meaningful that all other ${ }^{2} \Sigma^{+} R$ - levels which are observed to be markedly diffuse, i.e., $E(v=3), M(v=2)$ and $E(v=4)$, lie close to the levels $I(v=4,5$ and 6$)$. For these reasons the potential energy curve of the $\mathrm{A}^{\prime 2} \Sigma^{+}$state has been drawn in Fig. 3 on the assumption that the strong predissociations in ${ }^{2} \Sigma^{+} R$ - levels are caused through the intermediary of the $I^{2} \Sigma^{+}$state while the unperturbed wave functions of the ${ }^{2} \Sigma^{+} R-$ levels below $72000 \mathrm{~cm}^{-1}$ do not overlap strongly with $\mathrm{A}^{\prime}{ }^{2} \Sigma^{+}$. The assumed course of the $A^{\prime}$ potential curve is also in accordance with the observation that $I \sim A^{\prime}$ interactions are relatively weak for the higher vibrational levels of $I(v=4,5,6,7)$ whereas the lowest levels of $I$, for which the interaction with $A^{\prime}$ is expected to be very strong, are not observed. Eight R- states of symmetry ${ }^{2} \Sigma^{+}$have now been positively identified below the first ionization limit of NO. The assignment of these states as the first members of three Rydberg series is illustrated in Figs. la and 3. Arguments for the assignment of six of these states have been presented by Huber and Miescher (1963). The two states $\mathrm{R}^{2} \Sigma^{+}$and $\mathrm{Y}^{2} \Sigma^{+}$have been 
identified in the meantime. Their energy positions coincide precisely with the values found by extrapolation of the series initiated by $(\sigma 3 p) D^{2} \Sigma^{+}$and ( $\sigma 4 \mathrm{p}) \mathrm{M}^{2} \Sigma^{+}$. The ${ }^{2} \Sigma^{+}$states $\mathrm{D}, \mathrm{M}, \mathrm{R}$, and $\mathrm{Y}$ are related to the ${ }^{2} \Pi$ states $C, K, Q$, and W by "pure precession" (cf., Section III below). This relationship manifests itself in large $\Lambda$ - splittings in the ${ }^{2} \Pi$ states, from which constants $q$ are derived, whereas in the ${ }^{2} \Sigma^{\dagger}$ states the observed (apparent) B- values ( $B_{\text {app }}$ ) differ from true rotational constants ( ${ }_{\text {true }}$ ) according to $\mathrm{B}_{\text {app }}-\mathrm{B}_{\text {true }}= \pm \mathrm{q}$, where $\mathrm{q}$ is taken from the appropriate ${ }^{2} \Pi$ state. The positive sign applies for the p-complexes discussed here, because the $\Sigma$ states lie above the $I$ states. The $B$ - values for $R$ - states quoted in Table 1 are defined as $B(N)=\Delta_{1} F / 2(N+1)$, except for $H(v=0,1)$ where they are the $B_{\text {true }}$ values evaluated by Huber and Miescher (1963). Table 5 shows that the differences $B_{\text {app }}-B_{\text {true }}$ observed for $v=0$, with $B_{\text {true }}$ taken as $\mathrm{B}_{\mathrm{O}}\left(\mathrm{NO}^{+}, \mathrm{x}^{1} \Sigma^{+}\right)=1.992 \mathrm{~cm}^{-1}$, agree moderately well with the $\mathrm{q}$ values of the corresponding $\Pi$ states.

In the absorption spectrum, the transitions leading to the states D, $M, R, Y$ of configuration $\sigma \mathrm{np}$ appear with greater intensity than those assigned with excitation of $\sigma \mathrm{ns}$ or $\sigma \mathrm{nd}$. This might be the reason that the $\sigma \mathrm{np}$ series is observed up to higher members than the series $\sigma \mathrm{ns}$ and $\sigma$ nd. The absorption spectrum has been searched for signs of the states $\sigma 6 \mathrm{~s}, \sigma 7 \mathrm{~s}, \sigma 5 \mathrm{~d}$; however, due to overlapping with stronger bands or to low absorption intensity on the present plates no assignments could be made, with the possible exception of $\sigma 7 \mathrm{~s}$. Near the position expected for the $(0,0)$ transition of $(\sigma 7 s) z^{2} \Sigma^{+}-x^{2} \Pi$, a Rydberg band is clearly 
observed in the spectrum of $\mathrm{N}^{14} \mathrm{O}$. The lowest established rotational level in this state is the level with $N=1$ at $71588.9 \mathrm{~cm}^{-1}$. Presence or absence of a level $\mathrm{N}=0$ cannot be decided because of weakness of the absorption, but the state is almost certainly a ${ }^{2} \Sigma^{+}$state because it does not show a $\Lambda$ splitting while all ${ }^{2} \Pi R$ - states of NO show large $A$ - splittings even at low $\mathrm{J}$ - values. However, since the value of $\mathrm{B}=1.859 \mathrm{~cm}^{-1}$ is low for a $\mathrm{R}$ state with $\mathrm{v}=0$, and since no corresponding bands are found in the spectra of the other isotopes, the assignment $\sigma 7 \mathrm{~s}$ must be considered as tentative. Newly identified vibrational levels of already known ${ }^{2} \Sigma^{+}$states are included in Table 1. The levels $v=3,4$ of $(\sigma 4 s) \mathrm{E}^{2} \Sigma^{+}$are securely identified on the basis of vibrational isotope shifts. It is noteworthy that $\mathrm{E}-\mathrm{X}(3,0)$ is markedly diffuse in $\mathrm{N}^{14} \mathrm{O}$ but sharp in the other two isotopes, and $E-X(4,0)$ has almost disappeared on the high dispersion plates on account of diffuseness in $\mathrm{N}^{14} \mathrm{O}$, but can well be seen, though it is diffuse without line structure, in $\mathrm{N}^{15} \mathrm{O}$ and is only slightly diffuse in $\mathrm{NO}^{18}$. In accordance with these observations in the absorption spectrum is the fact that Feast (1950) has found no emission of bands with $v^{\prime} \geq 3$ in the E-A system of $\mathrm{N}^{14} \mathrm{O}$. As a curiosity it might be mentioned that in $\mathrm{NO}^{18}$ the lines of the 3,0 band of $E^{2} \Sigma^{+}-x^{2} \Pi$ lie very close to the corresponding lines of the 0,0 band of $N^{2} \Delta-x^{2} \Pi$ (to be described in Section IV), resulting in an accidental "doublet" structure with separations of 3 to $5 \mathrm{~cm}^{-1}$. The level $v=2$ of $(\sigma 5 p) M^{2} \Sigma^{+}$is found diffuse without line structure; the diffuseness in this case seems to increase from $N^{14} O$ to $N O^{18}$. M $-X$ bands with $v^{\prime}>2$ have not been found. Finally, the levels $v=5,6$ of $(\sigma 4 p) D^{2} \Sigma^{+}$ 
are entered into Table 1 (higher $\epsilon$-bands). Their appearance is related to a heterogeneous interaction with ${ }^{2} \Pi$ levels which will be described in a subsequent paper.

\section{III. ${ }^{2}$ II STATES}

As has already been stated the perturbations in ${ }^{2} \Pi$ states are complicated by the fact that above $64000 \mathrm{~cm}^{-1}$ three ${ }^{2} \overline{\Pi l} \mathrm{NR}$ - states with their numerous vibrational levels, and each with two widely separate spin doublet components, enter into competition with the ${ }^{2} \Pi R$ - states of the series $\pi$ np. The higher ${ }^{2} \Pi$ NR-states joining the low lying and familiar $B{ }^{2} \Pi$ state are designated $L^{2} \Pi$ and $P^{2} \Pi$. Weak bands of the $L(v)-X(0)$ progression begin to be visible for all three isotopes near $65300 \mathrm{~cm}^{-1}$ (see Table 2), whereas a lower member has been found for $\mathrm{N}^{14} \mathrm{O}$ only due to an interaction with the $R$ - state $K^{2} \Pi(v=0)$. Figures la and 3 show the $L$ state near $63000 \mathrm{~cm}^{-1}$, a position which is based on the isotope shifts found in the bands near $65300 \mathrm{~cm}^{-1}$. The $P^{2} \Pi$ state first appears as a strong band near 66000 $\mathrm{cm}^{-1}$ where it enters into a three-state interaction (to be described in a later paper) with $C^{2} \Pi(v=6)$ and $B^{2} \Pi(v=24)$.

All ${ }^{2} \Pi R$ - levels mixing with $B^{2} \Pi(v)$ below $64000 \mathrm{~cm}^{-1}$ belong to the state $\pi 3 \mathrm{p} \mathrm{C}^{2} \Pi\left(\mathrm{H}^{\prime 2} \Pi\right.$ of $\pi 3 \mathrm{~d}$ does not interact noticeably). Near the pair of levels $C(v=5)+B(v=21)$ the $R$ - state $\pi 4 p K^{2} \Pi$ enters into the picture with its $v=0$ level. While this level is only weakly perturbed, the $v=1$ level shows a pronounced interaction with $B(v=25)$.

The list of identified vibrational levels of $B^{2} \Pi$ is now complete and continuous up to $v=25$ near $66800 \mathrm{~cm}^{-1}$. Between 67000 and $72000 \mathrm{~cm}^{-1}$ 
no ${ }^{2} \Pi$ NR-bands have been found in $N^{14}$ O except where they coincide with and are perturbed by ${ }^{2} \Pi \mathbf{R}$-bands. The data available on NR-levels in this region are too incomplete to permit the evaluation of reliable deperturbed B-values, vibrational intervals or isotope shifts. For this reason, the assignment of the observed ${ }^{2} \Pi \mathrm{NR}$ - levels above $67000 \mathrm{~cm}^{-1}$ to specific electronic states $B^{2} \Pi, L^{2} \Pi$ or $P^{2} \Pi$ becomes increasingìy probiematical. The only ${ }^{2} \Pi$ bands found in the region 67000 to $68800 \mathrm{~cm}^{-1}$, apart from weak bands tentatively labeled $\beta(26,0)$ and $\beta(28,0)$ (cf., Table 2) are the bands observed in all three isotopes near $68600 \mathrm{~cm}^{-1}$. The study of the perturbed structure and isotope shifts of these bands shows that a vibrational level of a ${ }^{2} \Pi$ NR-state, probably $L^{2} \Pi(v=6)$, is mixed with a new $R$ - state which appears at this energy, namely $\pi 5 p Q^{2} \Pi(v=0)$. At somewhat higher energy $\left(69000 \mathrm{~cm}^{-1}\right)$ the $v=2$ level of $\mathrm{K}^{2} \Pi$ appears with great intensity. While no appreciable perturbation of this level arises in $\mathrm{N}^{15} \mathrm{O}$ and $\mathrm{NO}^{18}$, it is mixed in $\mathrm{N}^{14} \mathrm{O}$ with the close lying level $\mathrm{B}^{2} \Pi(v=29)$ at low J - values.

Above $69050 \mathrm{~cm}^{-1}$ the next ${ }^{2} \Pi$ bands are found in the region from 70600 to $71500 \mathrm{~cm}^{-1}$. The analyses of all of these bands yield perturbed upper state rotational term sequences resulting from the mixing of ${ }^{2} \Pi \mathrm{NR}$ and ${ }^{2} \Pi R$ - levels. The study of the perturbed $B(J)$ - values and of vibrational intervals leads to the identification of the new $R-$ state $\pi 6 \mathrm{p} \mathrm{W}^{2} \Pi$ $(v=0)$ as well as of the vibrational levels $Q^{2} \Pi(v=1)$ and $K^{2} \Pi(v=3)$. Mixed with these three $\mathrm{R}$ - levels are a number of NR-levels. A rigorous deperturbation of the observed rotational term sequences is not possible 
mainly because only one spin doublet component of each state has been observed in this region. Nevertheless an approximate deperturbation, ignoring the influence of the unobserved doublet components, can be carried out and the results are illustrated in Fig. 4. It is interesting that the observed terms can be interpreted as a superposition of the three $R$ - levels $W(v=0)$, $Q(v=1)$, and $K(v=3)$ onto a rather rapidly converging set of ${ }^{2} \Pi N R$ - levels with steadily decreasing B-values. It almost certainly is meaningful that this convergence of NR- levels is observed at an energy immediately below the lowest possible dissociation limit for excited ${ }^{2} \Pi$ states, i.e., the limit $N\left({ }^{2} D\right)+O\left(^{3} P\right)$ at $71660 \mathrm{~cm}^{-1}$. Although the relationship of the converging ${ }^{2} \Pi$ NR- levels to the three known ${ }^{2} \Pi$ NR- states cannot be determined from existing data, the isotope shift of the level BLP (d) interacting with $\mathrm{K}(\mathrm{v}=3)-$ assuming that we are dealing with the same NR- level in all three isotopes points to an electronic energy in the general vicinity of the $L^{2} \Pi$ and $P^{2} \Pi$ electronic states.

As has already been mentioned in Section II the newly identified $\pi \mathrm{np}$ states $Q^{2} \Pi$ and $w^{2} \Pi$, with $n=5$ and 6 respectively, are closely related to ${ }^{2} \Sigma^{+} R$ - states in exactly the same way as has been observed before for the lower members $n=3$ and 4 of the same Rydberg series. The data for all four pairs of $\Sigma-\Pi$ states are presented in Table 5. With increasing n the distance $\nu(\Sigma, \Pi)$ shows a regular decrease, accompanied by a corresponding increase of the constant $q$ of the $\Lambda$-doubling in agreement with the "pure precession" formula

$$
q=\frac{2 \mathrm{~B}^{2} \ell(\ell+1)}{\nu(\Sigma, \Pi)}
$$


with $\ell=1$. Thus the states of Table 5 form a good example of a Rydberg series of $p$-complexes. Related to the size of the $\Lambda$-doubling in the $I$ states is the excess of the observed "apparent" rotational constant B app of the $\Sigma$ states over the unperturbed Rydberg value (cf., Section II).

\section{IV. ${ }^{2} \Delta$ STATES}

The constants of the identified ${ }^{2} \Delta$ states are collected in Table 3. Prominent among these states is the long progression of vibrational levels belonging to the NR-state $B^{\prime}{ }^{2} \Delta$. Their lowest members have long been known from the emission spectrum of the NO molecule in which they appear as $\beta^{\prime}$ - bands $\left(B^{\prime 2} \Delta-X^{2} \Pi\right.$ ) in the vacuum ultraviolet (Miescher 1956) and as $B^{\prime 2} \Delta-B^{2} \Pi$-transitions in the visible region (Huber, Huber and Miescher 1963, Huber 1964). In the emission spectrum nearly all ${ }^{2} \Sigma$ and ZI levels above the dissociation limit of the ground state are missing because of predissociation by the repulsive $A^{\prime}{ }^{2} \Sigma^{+}$state, so that the ${ }^{2} \Delta$ levels are almost the only ones leading to emission at the higher energies. The vibrational levels of $B^{\prime 2} \Delta$ are now identified up to $v=10$. By successive analysis of these NR- levels it is possible to recognize Rlevels of ${ }^{2} \Delta$ symmetry by their interaction with the $B^{\prime}{ }^{2} \Delta N R$ - levels in a way which is exactly analogous to the situation encountered in the interactions between the vibrational levels of the $B^{2} \Pi N R$ - state and the various ${ }^{2} \Pi R$-states. In these interactions it is important to note that the NR - levels with high values of $v$ exhibit large isotope shifts in contrast to the low vibrational levels of the higher lying $R$ - states, such that the energy coincidences between $R$ - and NR- levels shift from isotope to isotope, 
leading to widely different appearance of the perturbations in the spectra of the different isotopes.

The levels $v=4,6,7$, and 10 of $\mathrm{B}^{\prime 2} \Delta$ are strongly perturbed in $\mathrm{N}^{14} \mathrm{O}^{16}$. The interaction in $\mathrm{v}=4$ is caused by the $\mathrm{R}-$ level $\mathrm{F}^{2} \Delta(\mathrm{v}=1)$ as has been stated before (Lagerquist and Miescher 1962). In $v=6$ a similar interaction occurs with $F^{2} \Delta(v=2)$, and although the $\beta^{\prime}(6,0)$ band of $\mathrm{N}^{14} \mathrm{O}^{16}$ is hidden in the strong band of $\mathrm{K}^{2} \Pi-X(1,0)$, the interaction parameter can be determined from the $B(J)$-curve of $F^{2} \Delta(v=2)$, and comparison with the $\beta^{\prime}(6,0)$ and $F-X(2,0)$ bands of $N^{15} \mathrm{O}$ and $\mathrm{NO}^{18}$ shows that in all three isotopes the interaction energy is of nearly the same size $\left(H=100 \mathrm{~cm}^{-1}\right)$. In contrast, the perturbation of $B^{\prime}{ }^{2}(v=7)$ is limited to $\mathrm{N}^{14} \mathrm{O}^{16}$ and to values of $\mathrm{J} \leq 10 \frac{1}{2}$ with a considerably smaller value of $\mathrm{H}\left(\mathrm{H} \approx 35 \mathrm{~cm}^{-1}\right)$. The band designated $\mathrm{B}^{\prime 2} \Delta-\mathrm{X}^{2} \Pi(7,0)$ has previously been labeled " $\rho$-band" (Huber and Miescher 1963). The B(J) value of $B^{\prime 2} \Delta(v=7)$ rises rapidly from a $N R$ - value of $1.35 \mathrm{~cm}^{-1}$ at the lowest $\mathrm{J}$ towards the typical $\mathrm{R}$ - value of $\mathrm{B}=2 \mathrm{~cm}^{-1}$, giving the corresponding band the appearance of a $R$ - band apart from the lowest few lines. In the spectra of $\mathrm{N}^{15} \mathrm{O}$ and $\mathrm{NO}^{18}$ the two interacting states are clearly recognized as two separate ${ }^{2} \Delta$ levels, viz., the $B^{\prime 2} \Delta(v=7)$ level plus a ${ }^{2} \Delta$ R-level. Since no level belonging to $F^{2} \Delta$ falls near $B^{\prime}{ }^{2} \Delta(v=7)$, this ${ }^{2} \Delta R$ - level must belong to a new state $N^{2} \Delta$. This is without any doubt to be interpreted as the Rydberg state $\delta 4 \mathrm{~d}$ of the series which starts with $\delta 3 d \mathrm{~F}^{2} \Delta$. This assignment is in accordance with the observed quantum defect $\left[\delta\left(4 \mathrm{~d} \mathrm{~N} \mathrm{~N}^{2} \Delta\right)=+0.07\right.$ as compared to $\delta\left(3 \mathrm{~d} \mathrm{~F}^{2} \Delta\right)=+$ 0.06], with the observed isotope shifts and with the clearly identified 
higher vibrational levels $N(v=1)$ and $N(v=2)$ in all three isotopes. The identification of the state $\delta 4 \mathrm{~d} \mathrm{~N}^{2} \Delta$ provides excellent confirmation of the interpretation given by M. Huber (1964) on the Rydberg states which appear in the emission spectrum in the visible region.

The level designated $\mathrm{B}^{\prime}(\mathrm{v}=7)$, which in $\mathrm{N}^{14} \mathrm{O}^{16}$ goes over into $N(v=0)$ with increasing rotation, should be designated $B^{i}(v=7) \rightarrow$ iv iv $\left.=0\right)$. The related interacting level $N(v=0) \rightarrow B^{\prime}(v=7)$ has not been found in the absorption spectrum, partly because the corresponding band is overlapped by the strong $(5,0)$-band of $G^{2} \Sigma^{-}-X^{2} \Pi$, partly because of anomalously low intensity (cf., Section VI). The identification of the $N(0,0) \rightarrow B^{\prime}(7,0)$ -band in the emission spectrum will be described in a separate communication.

Above the level $N^{2} \Delta(v=0)$ the levels $B^{\prime}{ }^{2} \Delta(v=8,9)$ appear as nearly unperturbed NR- levels and, lying about midway between these, $F^{2} \Delta(v=3)$ appears as an almost pure $R$ - type level. The band $\beta^{\prime}(10,0)$, however, is strongly perturbed, and in $\mathrm{NO}^{18}$, where the band is easily analyzed, the $B(J)$-value of the $B^{\prime 2} \Delta(v=10)$ level falls near $B=1.5 \mathrm{~cm}^{-1}$, i.e., the level represents a 1:1 mixture of $R$ - and NR-state. A new ${ }^{2} \Delta R$ state is clearly required to explain this observation. While the $v=1$ level of the $R$ - state $N^{2} \Delta$ lying $350 \mathrm{~cm}^{-1}$ below the $B^{\prime}(v=10)$ level shows some NR - mixing, this can account for only a small fraction of the R-contribution to the $B^{\prime}(v=10)$ level; furthermore no vibrational level of $F^{2} \Delta$ falls into this region. The new $R$ - state, $U^{2} \Delta(v=0)$ evidently is the $\delta 5 d R-$ state which is expected to fall into the region near $70150 \mathrm{~cm}^{-1}$ and which 
continues the Rydberg series $\delta$ nd whose lower members are $F^{2} \Delta$ and $\mathbf{N}^{2} \Delta$

The level designated $\mathrm{B}^{\prime}(\mathrm{v}=10)$ of $\mathrm{NO}^{18}$ goes over slowly, with increasing $\mathrm{J}$, into $\mathrm{U}(\mathrm{v}=0)$, and it can therefore be designated as $B^{\prime}(v=10) \rightarrow U(v=0)$. The related interacting state $U(v=0) \rightarrow B^{\prime}(v=10)$ has not yet been identified partly because of overlapping with the strong band of $G^{2} \Sigma^{-}-X^{2} \Pi(8,0)$ and partly because of anomalously low intensity (see Section VI).

\section{POTENTIAL ENERGY CURVES}

All ${ }^{2} \Sigma^{+},{ }^{2} \Pi$ and ${ }^{2} \Delta$ states now known or expected with certainty below the first ionization limit are illustrated by means of potential energy curves in Fig. 3. A word about the meaning of potential curves may be in order here because the study of NO has produced a wealth of information on the structure of states at and above the energies corresponding to intersections of potential curves, and also because the merits of intersecting versus nonintersecting potential curves in describing perturbed states have been subject to controversy as well as confusion in the literature. The type of curves chosen for presentation in Fig. 3 violates the noncrossing rule for states of identical symmetry, i.e., the curves correspond to a low order of approximation in which homogeneous inter actions are disregarded. Nevertheless these curves are useful because the regularly spaced vibration-rotation levels of the unperturbed electronic states represented by these potential curves are connected to the actually observed levels through perturbation theory in a straightforward manner. In principle it would be desirable to describe the actually observed 
levels directly, without prior deperturbation, as vibrational-rotational eigenvalues belonging to perturbed potential energy curves. Such higher approximation potential energy curves can be obtained by introducing $R$ NR interactions into the electronic eigenvalue problem; they do obey the noncrossing rule, but they are meaningful only as far as they represent the electronic energy eigenvalues of a hypothetical molecule with static nuclei. A description of the vibration-rotation structure of the perturbed states, however, is not possible based on such curves in any simple and hence useful way, except when the interaction energy is very large compared to the vibrational energy. The analysis of the perturbations encountered in the excited states of NO shows that the ensemble of the observed levels can be described in a reasonably simple way only when perturbation theory is introduced to the over-all electronic-vibrationalrotational levels individually. The interactions between levels of $B^{2} \Pi$ and $C^{2} I$ have been described on this basis in Part I (Lagerquist and Miescher 1958) where a detailed deperturbation of vibration-rotation levels has been carried out and has demonstrated the usefulness of this concept. Interaction parameters $\mathrm{H}$ are obtained which are nearly independent of rotational energy for any one pair of interacting vibronic levels, and their variation with vibrational energy is fully consistent with the expected dependence of $\mathrm{H}$ on the vibrational overlap between the unperturbed states.

\section{INTENSITIES}

It has been pointed out repeatedly that Rydberg series belonging to 
the first limit of ionization $T_{\infty}=74740 \mathrm{~cm}^{-1}$ are not readily recognized in the absorption spectrum of NO. The spectrum above $1200 \AA$ consists of a superposition of long vibrational progressions of $\mathrm{R}$ - as well as NR-type transitions, the vibrational intervals within these progressions are often irregular because of strong interactions between $R$ - and NR-states, and the intensities also are strongly influenced by these interactions. Marmo (1953) has published a plot of the NO absorption spectrum recorded at a rather low resolution (about $1 \AA$ ). His Fig. 4 shows high peaks of the absorption coefficient in the wavelength range $1500-1300 \AA$. Most of them correspond to diffuse bands with the upper levels $I^{2} \Sigma^{+}, G^{2} \Sigma^{-}, E^{2} \Sigma^{+}(v=3)$ and $M^{2} \Sigma^{+}(v=2)$ enhanced in appearance by the low resolution used. Even on the high resolution plates of the present study line broadening due to predissociation often varies considerably from $N^{14} \mathrm{O}$ to $\mathrm{N}^{15} \mathrm{O}$ to $\mathrm{NO}^{18}$, thus leading to great apparent intensity differences for one and the same transition in different isotope spectra.

In addition to the fluctuations of apparent intensities due to predissociation there are many cases of perturbed intensities due to the mixing of sharp levels. For example, in the plot of absorption coefficients published by Marmo (1953) the $\beta$-bands have an average intensity one order of magnitude below that of the very prominent $\delta$ - bands with the exception of $\beta(12,0)$ and $\beta(15,0)$ which are mixed with $\delta(2,0)$ and $\delta(3,0)$ respectively (Lagerqvist and Miescher, 1958) and which appear with two to three times greater intensity than normal. Similarly, many of the ${ }^{2} \Pi$ NR-bands above $68000 \mathrm{~cm}^{-1}$ listed in Table 2 derive probably the greater part of 
their intensity from mixing with close lying and inherently stronger ${ }^{2} \Pi$ R-transitions.

The pair of bands $\beta(15,0)$ and $\delta(3,0)$ is a particularly nice example of two very strongly mixed bands in which nearly identical rotational structures are accompanied by nearly identical absorption intensities [for a reproduction and for further details see Part $I$, for a photometric record see Bethke (1959)]. The study of many of the perturbed bands reported in Tables $1-3$ has shown, however, that the intensities of transitions to a pair of mixing states need not be of comparable magnitude at all even though in such a case of crossing of two unperturbed total energy curves W (cf., Fig. 2) some other properties of the two interacting states become identical, e.g., B-values and spin splitting. In Table 6 five examples are listed of perturbed states for which the crossing of unperturbed energies occurs at low $J$ values and for which only one of the two perturbed $W^{\prime}$ rotational term sequences has been observed in absorption. Examples of this observation of unequal intensities are given for all three isotopes and for all three classes of interactions $\Sigma \sim \Sigma, \Pi \sim \Pi$, and $\Delta \sim \Delta$. The notation (already used in Section IV) $Q 0 \rightarrow L 6$ refers to a ${ }^{2} \Pi$ state which for $J=\frac{1}{2}$ is mostly $Q^{2} \Pi(v=0)$ [large $B$ - value] and which passes into mostly being $L^{2} \Pi(v=6)$ [small B-value] with increasing J. The interacting partner in this case is a level $L 6 \rightarrow Q 0$, however, for this and for all other examples listed in Table 6, the existence of the interacting partner not shown in the Table can only be deduced from a quantitative analysis of the observed interaction partner whereas direct observation of these missing states has not been possible. 
The interpretation of the absorption spectrum as given in Tables 1 3 and a satisfactory quantitative understanding of the observed states with their rotational structures depend crucially on the postulated existence of such states which are needed as interaction partners, but which due to a small transition probability are not easily observed in transitions from the ground state $x^{2} \Pi(v=0)$ of the molecule.

The theory of intensities in perturbations has been formulated by Dieke (1941) and it is fully consistent with the unequal intensities in pairs of mutually perturbed bands as observed in NO. Dieke has shown that for homogeneous perturbations (mixing of states with equal $\Lambda$ ) the perturbed intensities $I_{a}, I_{b}$ are related to the unperturbed intensities $I_{1}, I_{2}$ according to the equations

$$
\begin{aligned}
& \left(H^{2}+w^{2}\right) I_{a}=\left(H \sqrt{I_{1}} \mp w \sqrt{I_{2}}\right)^{2} \\
& \left(H^{2}+w^{2}\right) I_{b}=\left(w \sqrt{I_{1}} \pm H \sqrt{I_{2}}\right)^{2}
\end{aligned}
$$

The energy shifts $H$ (at the point of intersection, $J_{0}$ ) and $w$ (at the $J-$ value under consideration) are explained in Fig. 2. One of the perturbed amplitudes is a sum, the other a difference between terms containing the unperturbed amplitudes. It is immediately evident that one of the perturbed amplitudes must vanish when $I_{1} / I_{2}=w^{2} / H^{2}$. When the unperturbed intensities are of comparable magnitude $\left[I_{1} / I_{2} \approx 1\right]$, then one of the perturbed intensities becomes very small in the region of the crossing where $w^{2}=H^{2}$. Such a situation is illustrated in the schematic representation of one-dimensional wave functions in Fig. 5 . In the example shown, the 
wave function $\phi_{a}$ is large throughout the region in which wave function $\phi_{b}$ has an additional node. It is easily visualized that transitions to the states $a$ and $b$ from a third state $X$ whose wave function $\phi_{X}$ is localized in the same region will have very different probabilities. Thus the formulae allow for the observation of only one of two interacting states when the two unperturbed intensities are of comparabie măgnitude. On the other hand the equality of perturbed intensities found for the pair of bands $\delta(3,0)$ and $\beta(15,0)$ in the region where $\mathrm{w} \approx \mathrm{H}$ is consistent with the large observed difference between the unperturbed intensities $I(\delta) \gg I(\beta)$.

The thoughts outlined above can also be applied to radiationless transitions and they may be partly responsible for the wide range of probabilities for predissociation encountered in the $I^{2} \Sigma^{+}-\mathrm{X}^{2} \Pi$ system. For example, it may be significant that the two sharp observed vibrational levels of the $I^{2} \Sigma^{+}$state coincide closely with ${ }^{2} \Sigma^{+} \mathrm{R}$ - states, namely, $\mathrm{R}^{2} \Sigma^{+}(\mathrm{v}=0)$ in $\mathrm{N}^{15} \mathrm{O}$ and $\mathrm{E}^{2} \Sigma^{+}(\mathrm{v}=4)$ in $\mathrm{N}^{14} \mathrm{O}$; the overlap between these levels of $I$ with the repulsive $\mathrm{A}^{\prime}{ }^{2} \Sigma^{+}$state may be reduced through the interaction with $\mathrm{R}$ - states.

\section{IONIZATION POTENTIAL}

The four observed states of series $\sigma n p^{2} \Sigma^{+}$are free from detectable perturbations at low $\mathrm{J}$ - values, in $\mathrm{N}^{14} \mathrm{O}^{16}$. The observed term values $\mathrm{T}_{0}(\mathrm{n})=\mathrm{T}\left(\sigma \mathrm{np}{ }^{2} \Sigma^{+}, \mathrm{v}=0, \mathrm{~N}=0\right)$ can be fitted to the Rydberg formula

$$
T_{0}(n)=T_{0}(\infty)-\frac{109737 \mathrm{~cm}^{-1}}{(n-\delta)^{2}} .
$$

The series limit $T_{0}(\infty)$ obtained from this equation is equal to the first 
ionization potential of NO provided that the Rydberg states used are not strongly perturbed, and depending on the assumed variation of the quantum defect $\delta$ with increasing quantum number $n$. Since no constant value of $\delta$ represents more than two of the observed terms, we assume that the most probable value of $I(N O)$ is obtained using the highest two members with $n=5$ and 6 , which yield the value

$$
I\left(N O, x^{2} \Pi_{\frac{1}{2}}\right)=9.266 \pm 0.008 \mathrm{eV}\left(74740 \pm 60 \mathrm{~cm}^{-1}\right)
$$

The errors quoted here are based on a deviation of \pm 0.020 allowed between the values of $\delta(n=5)$ and $\delta(n=6)$. The term values used and the resultant quantum defects are listed in Table 7. The value of I(NO) derived here refers to the position of $\mathrm{NO}^{+},{ }^{1} \Sigma^{+}, \mathrm{v}=0, \mathrm{~J}=0$ measured relative to NO, $\mathrm{x}^{2} \Pi_{\frac{1}{2}}, v=0, \mathrm{~J}=\frac{1}{2}$. The same quantity has been de termined recently by Huber (1961), based on a Rydberg extrapolation of bands with unresolved rotational structure near $700 \AA$, with convergence towards the $\mathrm{A}^{1} \Pi$ state of $\mathrm{NO}^{+}$. His result is $\mathrm{I}\left(\mathrm{NO}, \mathrm{X}^{2} \Pi_{\frac{1}{2}}\right)=9.267 \pm$ $0.005 \mathrm{eV}$.

A closely related quantity has been determined by Watanabe (1954), and more recently by Nicholson (1963), i.e., the threshold for photoionization in NO gas at room temperature. Their threshold measurements are $9.25 \pm 0.02 \mathrm{eV}$ and $9.250 \pm 0.005 \mathrm{eV}$, respectively. These room temperature threshold energies should be $0.018 \mathrm{eV}$ lower than the spectroscopically determined ionization potential of the rotationless ground state ${ }^{2} \Pi_{\frac{1}{2}}$, because 1) at room temperature the long-wavelength onset of any absorption in NO is produced by the ${ }^{2} \Pi_{3 / 2}$ spin component whose energy 
is $0.015 \mathrm{eV}$ above ${ }^{2} \Pi_{\frac{1}{2}}$, and 2) an additional small reduction in threshold energy results from the population of rotational levels. The rotational structure gives rise to a sharp long-wavelength edge at $0.003 \mathrm{eV}$ below the rotationless energy, formed by the head of the branch with $\Delta J=-3 / 2$, assuming that the selection rule for $\Delta J$ in ionization of the $\bar{\pi} 2 \mathrm{p}$ electron is $\Delta \mathrm{J}= \pm 1 / 2, \pm 3 / 2$. The threshold energy of $9.248 \mathrm{eV}$ derived from our spectroscopic data is in excellent agreement with the direct measurements.

\section{CONCLUDING REMARKS}

The results of the investigation presented in this paper show that the extremely complex absorption spectrum of the NO molecule has now been analyzed almost up to the energy corresponding to the first ionization limit. All observed bands, most of which are highly perturbed, could be assigned to vibrational levels of either Rydberg- or of valence shell electronic states predicted from the electronic structure of the molecule. Es sential to the dissentangling of the overlapping band structures was the cooling of the NO gas to liquid oxygen temperature in order to shorten the individual bands, and also the study of the bands in three different isotopes. The data for the 70 energy levels given in Tables $1-3$ are well established. They are provisional only in the sense that more details regarding the observed rotational term sequences and details of the many interactions remain to be outlined in future papers.

NO should now be the molecule for which the variety of excited states below the first ionization limit is understood in most detail. It has become evident also that the many configuration interactions play an important 
part. Yet the system of NO states is a relatively simple one owing to the widely different properties of $R$-versus NR- levels. In other molecules such as $\mathrm{N}_{2}$ or $\mathrm{O}_{2}$ similar situations will perhaps be encountered although more serious complications will no doubt be found, since these molecules are not distinguished by a closed shell ${ }^{1} \Sigma^{+}$core in their $R$ - states.

It is planned to continue this research by photographing the NO spectrum at wavelengths shorter than $1400 \AA$ with high resolution shortly, and new spectrograms to be obtained at longer wavelengths should provide additional information which is needed to carry out at least a semiquantitative deperturbation primarily of the ${ }^{2} \Pi$ state ensemble.

\section{ACKNOWLEDGMENTS}

Again, as is true for all papers in this series, foremost thanks are due to Dr. G. Herzberg and Dr. A. E. Douglas (National Research Council of Canada) whose kind hospitality gave one of the authors (E. M.) the opportunity to use the NRC 10 -meter vacuum grating instrument. We thank Mr. F. Alberti for his expert help in the experiments and we acknowledge the participation of Prof. A. Lagerqvist and Prof. A. Lofthus in the meas urements and analyses. Collaboration of one of us (K.D.) in the NO project was first made possible by the financial support of the Swiss National Fund and was sponsored in part by the National Aeronautics and Space Administration under Grant NsG-414. The research was sponsored in part by the Air Force Cambridge Research Laboratories OAR through the European Office of Aerospace Research, United States Air Force, under Grant No. AF-EOAR 63-109. 


\section{REFERENCES}

Barrow, R. F., and Miescher, E. 1957, Proc. Phys. Soc. London $\underline{A} \underline{70}$, 219.

Bethke, G. W. 1959, J. Chem. Phys. 31, 662.

Dieke, G. H. 1941, Phys. Rev. 60, 523.

Feast, M. W. 1950, Can. J. Research $\underline{A}$ 28, 488.

Huber, K. P. 1961, Helv. Phys. Acta 34, 929.

, Huber, M., and Miescher, E. 1963, Phys. Letters (Netherlands)

3. 315 .

—, and Miescher, E. 1963, Helv. Phys. Acta 36, 257 (Part III).

Huber, M. 1964, Helv. Phys. Acta 37, 0000.

Lagerquist, A., and Miescher, E. 1958, Helv. Phys. Acta 31, 221 (Part I).

1962, Can. J. Phys. 40, 352 (Part II).

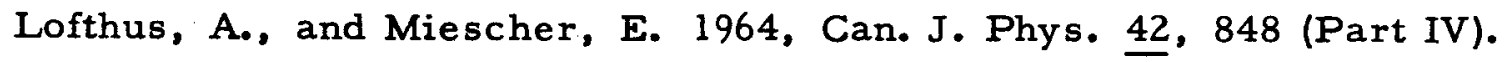

Marmo, F. F. 1953, J. Opt. Soc. Am. 43, 1186.

Miescher, E. 1956, Helv. Phys. Acta 29, 401.

Nicholson, A. J. C. 1963, J. Chem. Phys. 39, 954.

Watanabe, K. 1954, J. Chem. Phys. 22, 1564. 


\section{Table $1{ }^{2} \Sigma^{+}$-states}

$T \quad$ - Energy $\left(\mathrm{cm}^{-1}\right)$ of level $N=0, J=\frac{1}{2}$, relative to $\mathrm{X}^{2} \Pi\left(\mathrm{v}=0, J=\frac{1}{2}\right)$.

- Blank spaces indicate bands which are either not observed (too weak or overlapped) or not measured.

h - Head.

d - Diffuse lines:

vd - Very diffuse without line structure.

vvd - Maximum of continuous absorption.

* - Provisional value.

$+\quad$ - Value reported by Feast (1950).

Table $2^{2}$ II-states

$T$ - Energy $\left(\mathrm{cm}^{-1}\right)$ of level $N=1, J=\frac{1}{2}\left(J=1 \frac{1}{2}\right.$ for inverted levels) relative to $\mathrm{X}^{2} \Pi\left(v=0, J=\frac{1}{2}\right)$.

A - Spin doublet splitting $\left(\mathrm{cm}^{-1}\right)$.

B - Varying B-values $\left(\mathrm{cm}^{-1}\right)$ are given in two entries, the first for low $\mathrm{J}$, the second for highest $J$ observed.

$q \quad-\Lambda$-doubling [coefficient of $\mathrm{N}(\mathrm{N}+1)]$.

BLP- Mixed levels originating from $B^{2} \Pi, L^{2} \Pi, P^{2} \Pi$, with mixing ratios not yet determined.

$a, b, c, d, e$ - Vibrational quantum numbers not yet determined.

] - Brackets connect mutually interacting levels.

* - Provisional value.

$+\quad$ - Ir regular $\Lambda$-splittings are observed.

$\alpha \quad$ - See Huber and Miescher (1963). 
Table 1 ${ }^{2} \Sigma^{+}$-states

\begin{tabular}{|c|c|c|c|c|c|c|c|c|c|}
\hline \multicolumn{4}{|c|}{${ }^{2} \Sigma^{+}$states } & \multicolumn{2}{|c|}{$N^{14} o^{16}$} & \multicolumn{2}{|c|}{$\mathrm{N}^{15} \mathrm{O}^{16}$} & \multicolumn{2}{|c|}{$\mathrm{N}^{14} \mathrm{O}^{18}$} \\
\hline \multicolumn{2}{|c|}{$\mathbf{R}$} & NR & $\mathbf{v}$ & $\mathrm{T}$ & B & $T$ & B & $\mathrm{T}$ & B \\
\hline \multirow{3}{*}{$7 s$} & $S$ & & 2 & 71792 & 1.93 & 71707 & 1.867 & - & - \\
\hline & z & & 0 & 71587 & 1.859 & - & - & - & - \\
\hline & & I & 7 & $70938 \mathrm{~h}$ vd & 1.3 & - & - & - & - \\
\hline $6 p$ & $\mathrm{Y}$ & & 0 & 70846 & 2.11 & - & - & - & - \\
\hline \multirow[t]{3}{*}{$5 d$} & $\mathrm{v}$ & & 0 & \multicolumn{5}{|c|}{ (expected $\sim 70250 \mathrm{~cm}^{-1}$, not identified) } & \\
\hline & & I & 6 & 69990 & 1.350 & - & - & - & - \\
\hline & $\mathbf{E}$ & & 4 & - & - & $69870 \mathrm{~h}$ vd & - & $69792 \mathrm{~h} \mathrm{~d}$ & 1.78 \\
\hline \multirow[t]{4}{*}{$6 s$} & $\mathrm{~T}$ & & 0 & \multicolumn{5}{|c|}{ (expected $\sim 69870 \mathrm{~cm}^{-1}$, not identified) } & \\
\hline & $\mathrm{s}$ & & 1 & 69479.7 & 1.950 & 69433.8 & 1.880 & 69412.0 & 1.846 \\
\hline & M & & 2 & $69246 \mathrm{vd}$ & - & $69170 \mathrm{vvd}$ & - & $69120 \mathrm{vvd}$ & - \\
\hline & & I & 5 & $68928 \mathrm{~h}$ vd & 1.4 & $68831 \mathrm{~h}$ & $1.9 \rightarrow 1.3$ & $68790 \mathrm{~h}$ vd & - \\
\hline $5 p$ & $\mathbf{R}$ & & 0 & 68829.4 & 2.04 & 68821.3 & $1.9 \rightarrow 1.3$ & - & - \\
\hline \multirow[t]{4}{*}{$4 d$} & 0 & & 0 & 67810 & - & 67810 & - & 67810 & - \\
\hline & & I & 4 & - & - & $67835 \mathrm{~h} \mathrm{vd}$ & - & $67795 \mathrm{~h} \mathrm{vd}$ & 1.4 \\
\hline & $\mathbf{E}$ & & 3 & $67790 \mathrm{~d}$ & 1.91 & 67668 & 1.86 & 67607 & 1.82 \\
\hline & $\mathrm{H}$ & & 2 & $67360 \mathrm{~h}$ & - & - & - & - & - \\
\hline \multirow[t]{5}{*}{$5 s$} & $\mathbf{S}$ & & 0 & 67135.0 & 1.970 & & & & \\
\hline & $\mathbf{M}$ & & 1 & 66972 & 1.995 & 66929 & 1.90 & 66906 & 1.87 \\
\hline & $\mathrm{D}$ & & 6 & $66000 *$ & $1.77 *$ & $65750^{*}$ & - & - & - \\
\hline & $\mathbf{E}$ & & 2 & $65514.9+$ & $1.9408+$ & 65428.8 & 1.874 & 65388.6 & 1.842 \\
\hline & $\mathrm{H}$ & & 1 & 65044.9 & 1.976 & 65000 & - & - & - \\
\hline \multirow[t]{3}{*}{$4 p$} & $\mathbf{M}$ & & 0 & 64659 & 2.013 & 64655 & 1.95 & 64653 & 1.91 \\
\hline & $\mathrm{D}$ & & 5 & $64150 *$ & - & $63960 *$ & - & $63872 *$ & - \\
\hline & $\mathbf{E}$ & & 1 & $63204.7+$ & $1.9591+$ & - & - & - & - \\
\hline \multirow[t]{2}{*}{$3 d$} & $\mathrm{H}$ & & 0 & 62705.5 & 1.994 & 62701 & - & - & - \\
\hline & $\mathrm{D}$ & & 4 & 62107.2 & 1.9047 & 61956.1 & 1.8383 & - & - \\
\hline $4 s$ & $\boldsymbol{E}$ & & 0 & $60862.8+$ & $1.9772+$ & - & - & - & - \\
\hline
\end{tabular}




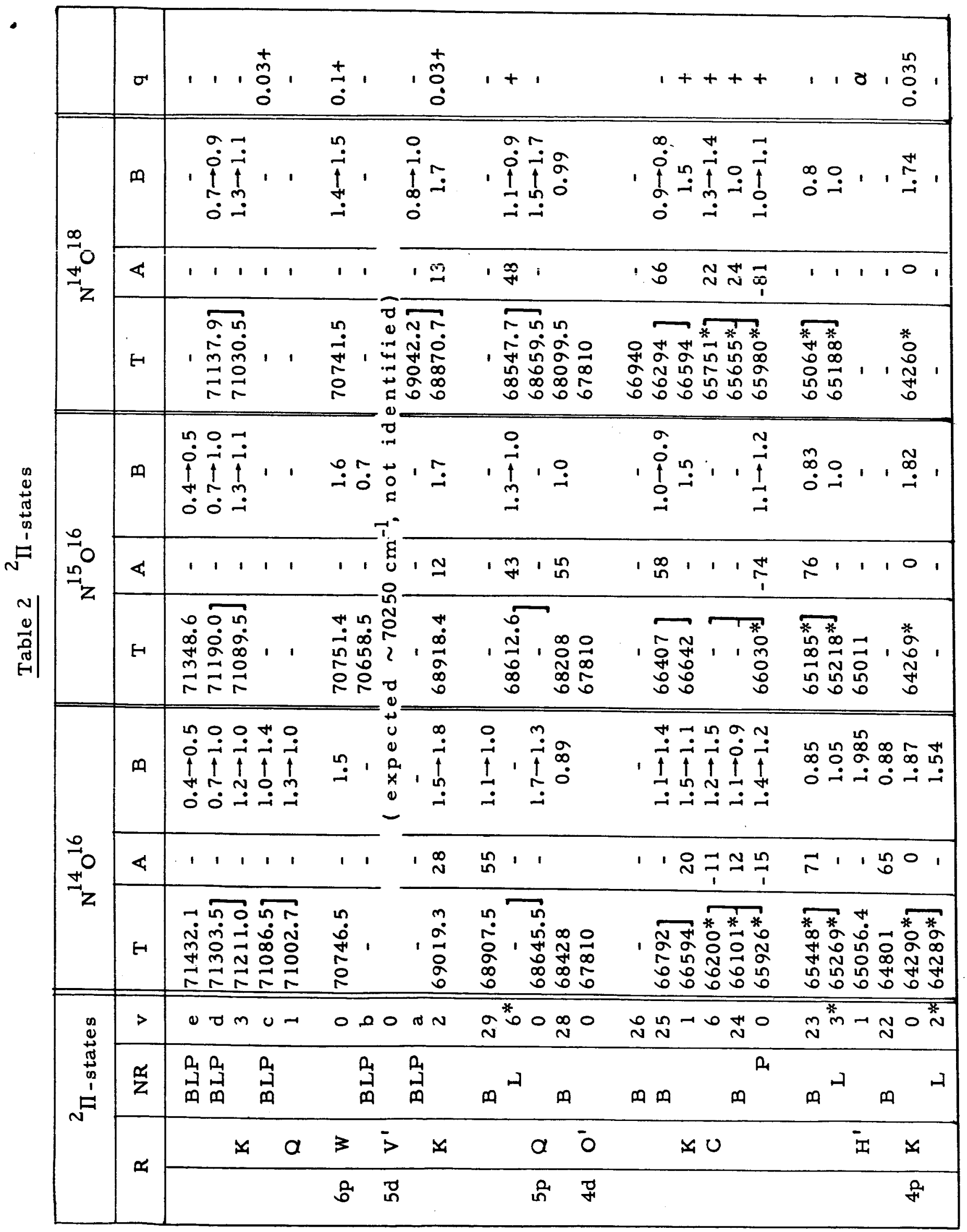


Table $3{ }^{2} \Delta$-states

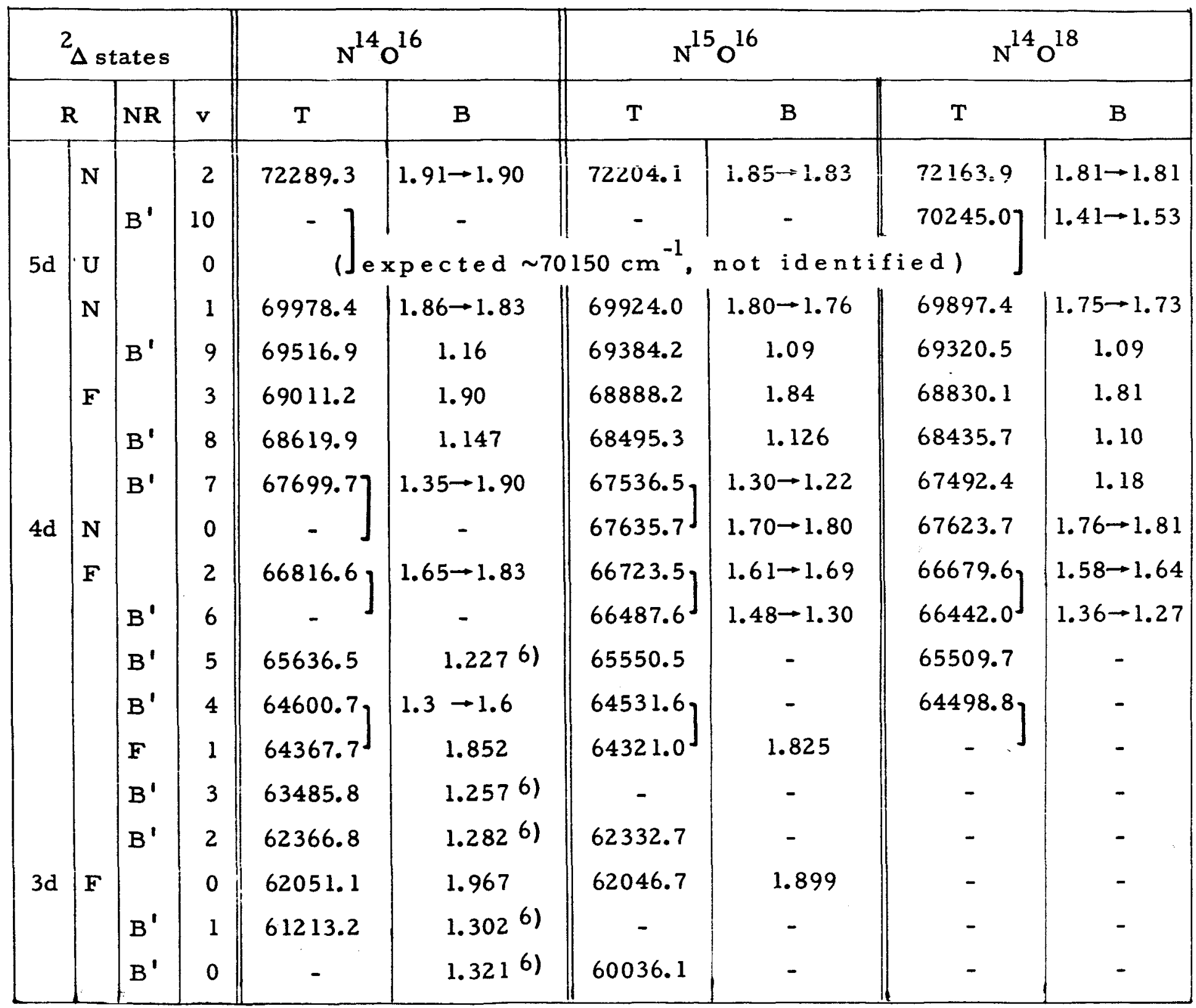

$T-$ Energy of level $N=2, J=1 \frac{1}{2}$, relative to $\left.x^{2} \Pi \quad(v=0), J=\frac{1}{2}\right)$.

All $B^{\prime}{ }^{2} \Delta$ levels show a small inverted doublet splitting (cf., Miescher, 1956 ). 
Table $4 I^{2} \Sigma^{+}-x^{2} \Pi$ - bands

\begin{tabular}{|c|c|c|c|c|c|c|c|c|c|}
\hline & \multicolumn{3}{|c|}{$\mathrm{N}^{14} \mathrm{o}^{16}$} & \multicolumn{3}{|c|}{$N^{15} o^{16}$} & \multicolumn{3}{|c|}{$N^{14} o^{18}$} \\
\hline $\mathrm{v}^{*}$ & $\nu_{h}$ & $\Delta G$ & B & $\nu_{h}$ & $\Delta \mathrm{G}$ & B & $\nu_{h}$ & $\Delta G$ & B \\
\hline 0 & \multicolumn{9}{|c|}{$T_{0} \approx 63000 \pm \mathrm{cm}^{-1}$} \\
\hline 4 & - & & & $67835 \mathrm{~d}$ & & - & $67795 \mathrm{~d}$ & & 1.4 \\
\hline 5 & $68928 \mathrm{~d}$ & & 1.38 & $68831 \mathrm{~s}$ & 996 & $(1.9) \rightarrow 1.3$ & $68790 \mathrm{~d}$ & 995 & - \\
\hline 6 & $69993 \mathrm{~s}$ & 1065 & 1.350 & - & & & & & \\
\hline 7 & $70935 \mathrm{~d}$ & 945 & 1.32 & - & & & & & \\
\hline
\end{tabular}

$\nu_{h}-R_{11}$-head.

d - Diffuse.

s - Sharp.

$\mathrm{v}^{*}$ - Tentative vibrational quantum number.

Table 5 p-complexes

\begin{tabular}{|c|c|c|c|c|c|}
\hline & & & \multicolumn{2}{|c|}{${ }^{2} \Pi$} & ${ }^{2} \Sigma^{+}$ \\
$\mathrm{n}$ & $\mathrm{np}-$ complex & $\Delta \nu(\Sigma, \Pi)$ & $\mathrm{q}_{\text {calc }}$ & $\mathrm{q}_{\text {obs }}$ & $\mathrm{B}_{\text {app }}{ }^{-\mathrm{B}_{\text {true }}}$ \\
\hline 6 & $\mathrm{Y}^{2} \Sigma^{+}+\mathrm{W}^{2} \Pi$ & 100 & 0.16 & 0.1 & .12 \\
5 & $\mathrm{R}^{2} \Sigma^{+}+\mathrm{Q}^{2} \Pi$ & 184 & 0.087 & 0.03 & .05 \\
4 & $\mathrm{M}^{2} \Sigma^{+}+\mathrm{K}^{2} \Pi$ & 370 & 0.043 & 0.035 & .02 \\
3 & $\mathrm{D}^{2} \Sigma^{+}+\mathrm{C}^{2} \Pi$ & 920 & 0.017 & 0.016 & .00 \\
\hline
\end{tabular}

n - Principal quantum number.

$\Delta \nu(\Sigma, \Pi)$ - Energy difference between $\Sigma$ and $\Pi$ state of the complex. $\mathrm{B}_{\text {true }}$ has been adopted identical to $\mathrm{B}_{\mathrm{O}}\left(\mathrm{NO}^{+}, \mathrm{X}^{1} \Sigma^{+}\right)=1.992 \mathrm{~cm}^{-1}$. 
Table 6 Mixed levels with anomalous absorption intensities

\begin{tabular}{|c|c|c|c|}
\hline Isotope & ${ }^{2} \Sigma^{+} \rightarrow^{2} \Sigma^{+}$ & ${ }^{2} \Pi \rightarrow{ }^{2} \Pi$ & ${ }^{2} \rightarrow^{2} \Delta$ \\
\hline$N^{14} O^{16}$ & & $\begin{array}{l}Q(v=0) \rightarrow L(v=6) \\
W(v=0) \rightarrow B L P(v=b)\end{array}$ & $B^{\prime}(v=7) \rightarrow N(v=0)$ \\
\hline$N^{15} O^{16}$ & $R(v=0) \rightarrow I(v=5)$ & & \\
\hline$N^{14} O^{18}$ & & & $B^{\prime}(v=10) \rightarrow U(v=0)$ \\
\hline
\end{tabular}

Table 7 Quantum defects in the series $(\sigma \mathrm{np})^{2} \Sigma^{+}$

\begin{tabular}{|c|c|l|c|}
\hline $\mathrm{n}$ & State & $\mathrm{T}_{\mathrm{O}}(\mathrm{n})$ & $\delta(\mathrm{n})$ \\
\hline$\infty$ & $\mathrm{NO}^{+} \mathrm{X}^{1} \Sigma^{+}$ & $74740 \mathrm{~cm}^{-1}$ & - \\
6 & $\mathrm{NO} \mathrm{Y}^{2} \Sigma^{+}$ & 70846 & 0.691 \\
5 & $\mathrm{R}^{2} \Sigma^{+}$ & 68829 & 0.691 \\
4 & $\mathrm{M}^{2} \Sigma^{+}$ & 64659 & 0.701 \\
3 & $\mathrm{D}^{2} \Sigma^{+}$ & 53291 & 0.738 \\
\hline
\end{tabular}




\section{FIGURE CAPTIONS}

Figure 1 a) Electronic energy level diagram; b) Schematic potential energy curves.

Figüre 2 Unperturbed and perturbed rotational term sequences.

Figure 3 Potential energy curves for ${ }^{2} \Sigma^{+},{ }^{2} 11,{ }^{2} \Delta$-states.

Figure $4{ }^{2} \Pi$ levels $\left(N^{14} O^{16}\right)$ near dissociation limit $N\left({ }^{2} D\right)+O\left({ }^{3} P\right)$ at $71660 \mathrm{~cm}^{-1}$. Dots connected with heavy curves represent observed energy levels. An approximate deperturbation of the interacting pairs $Q(v=1)+B L P(c)$ and $K(v=3)+B L P(d)$ yields the light lines. $B$-values are noted on the right-hand side.

Figure 5 Unperturbed and perturbed vibrational wave functions. 


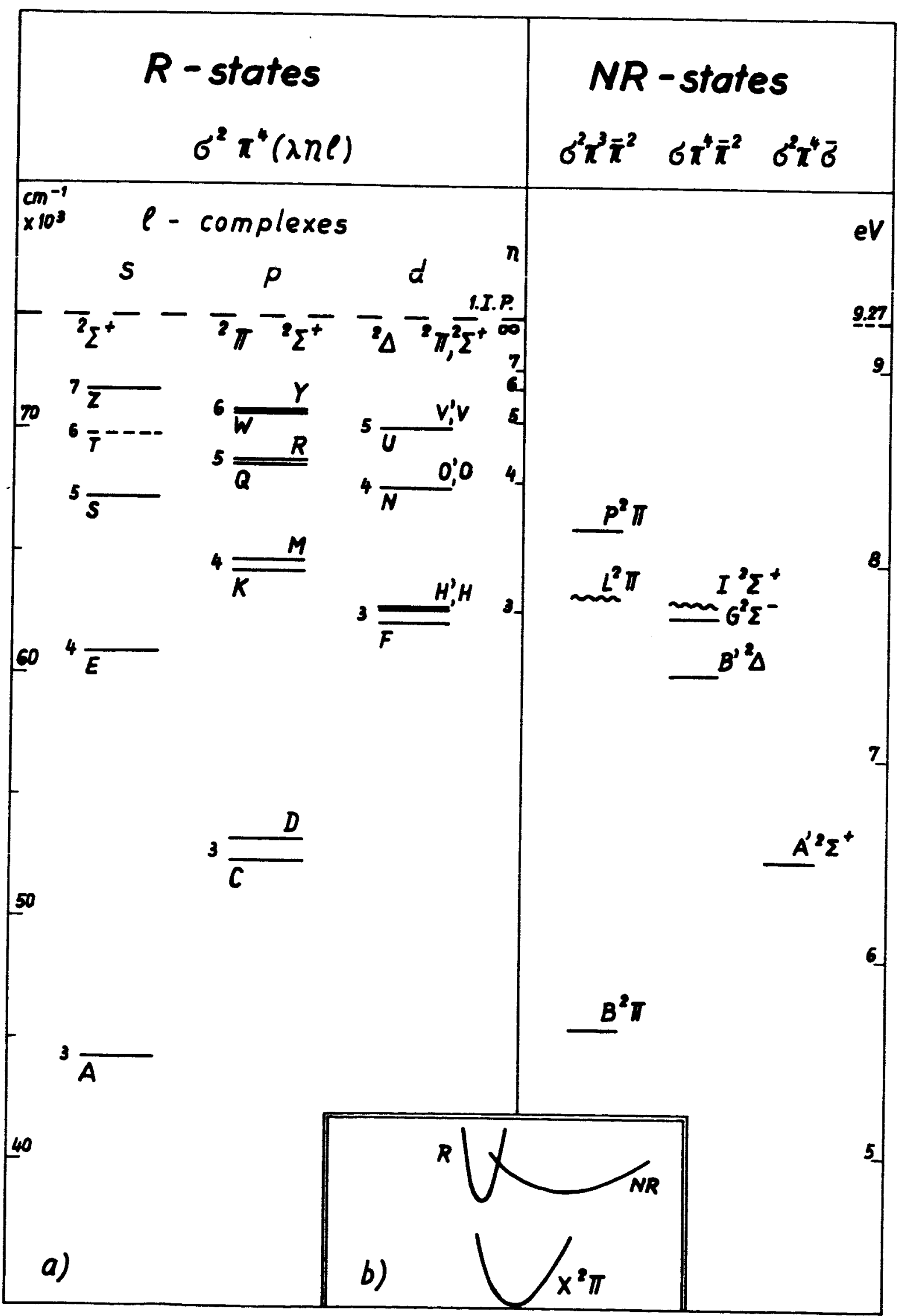




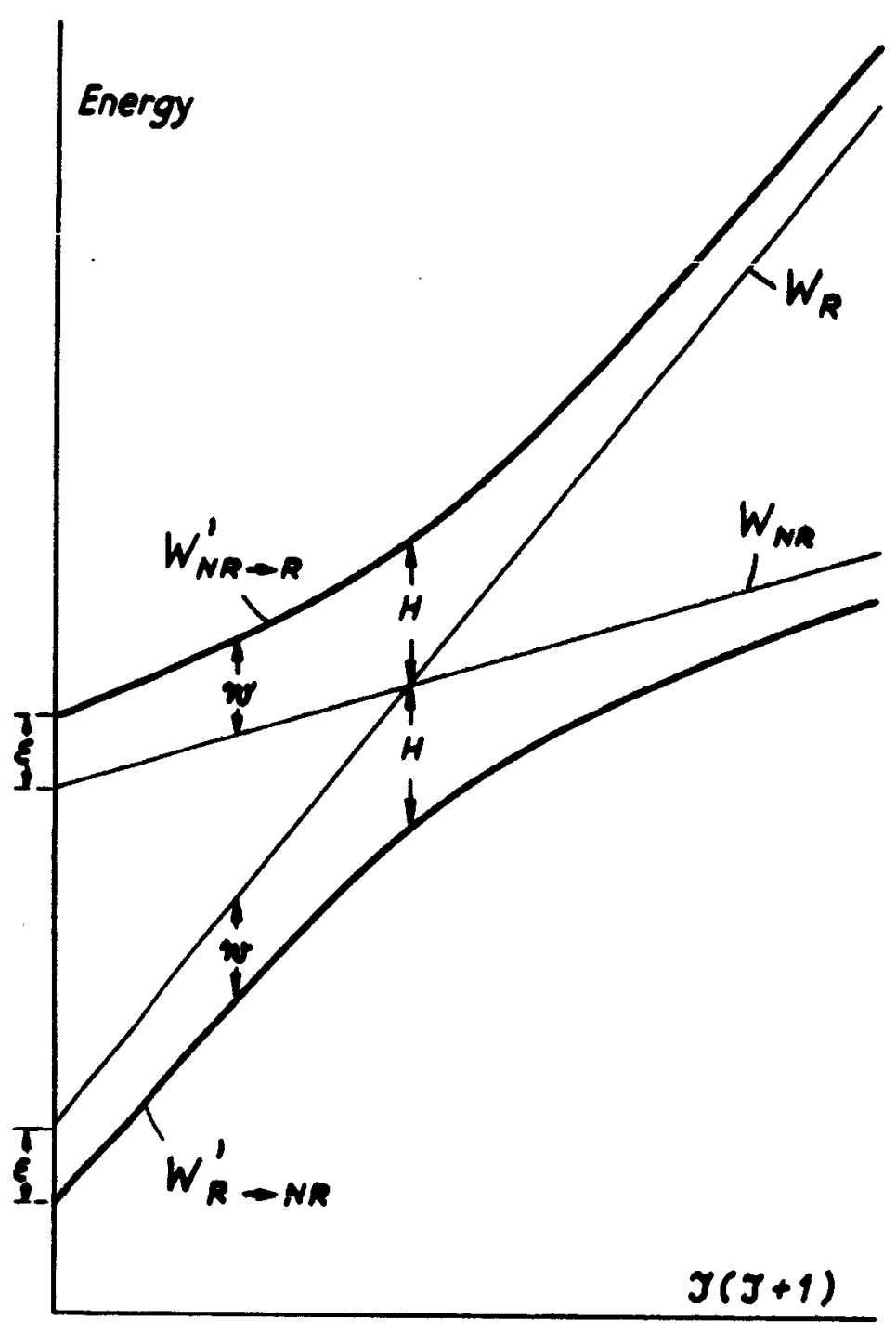




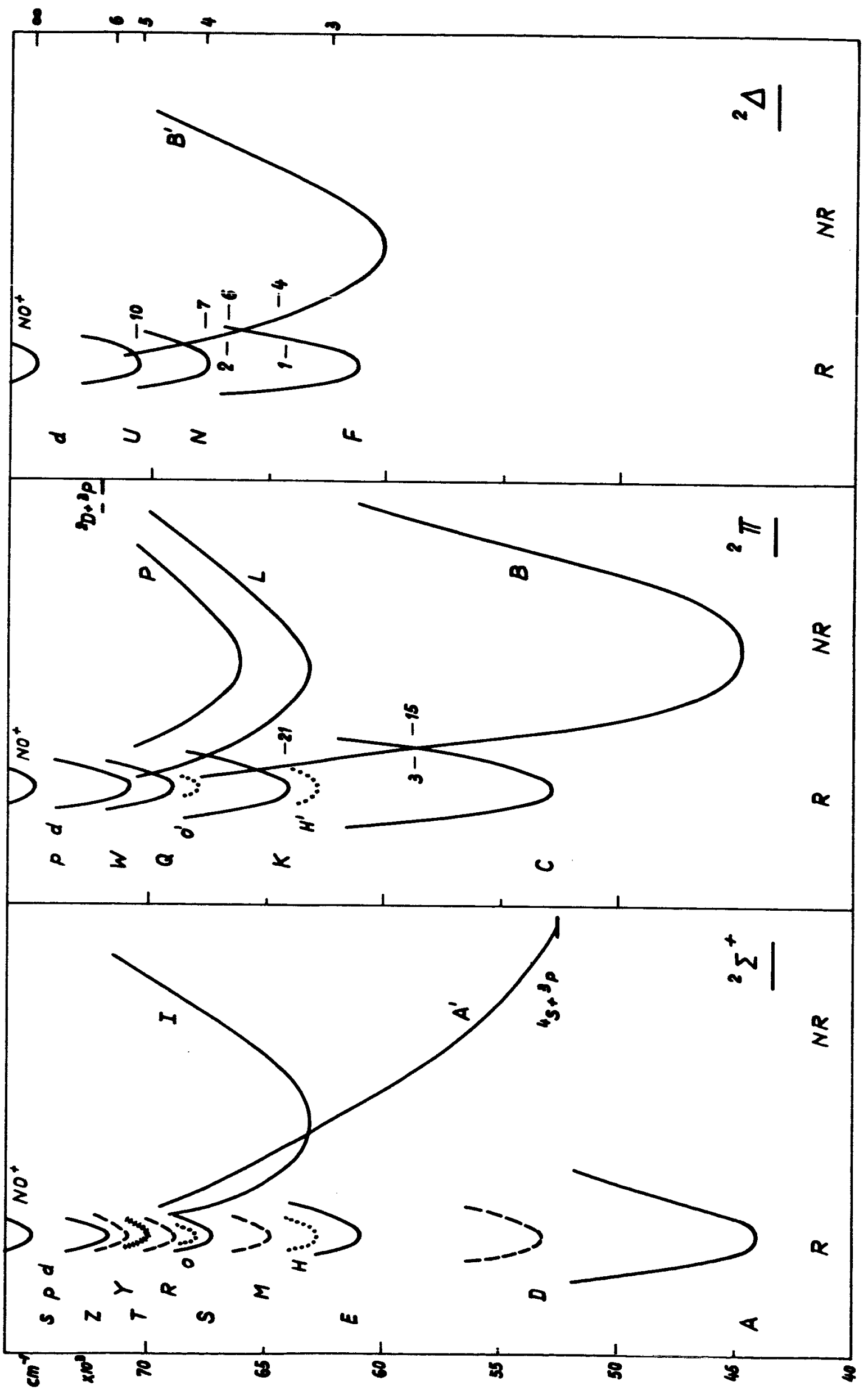




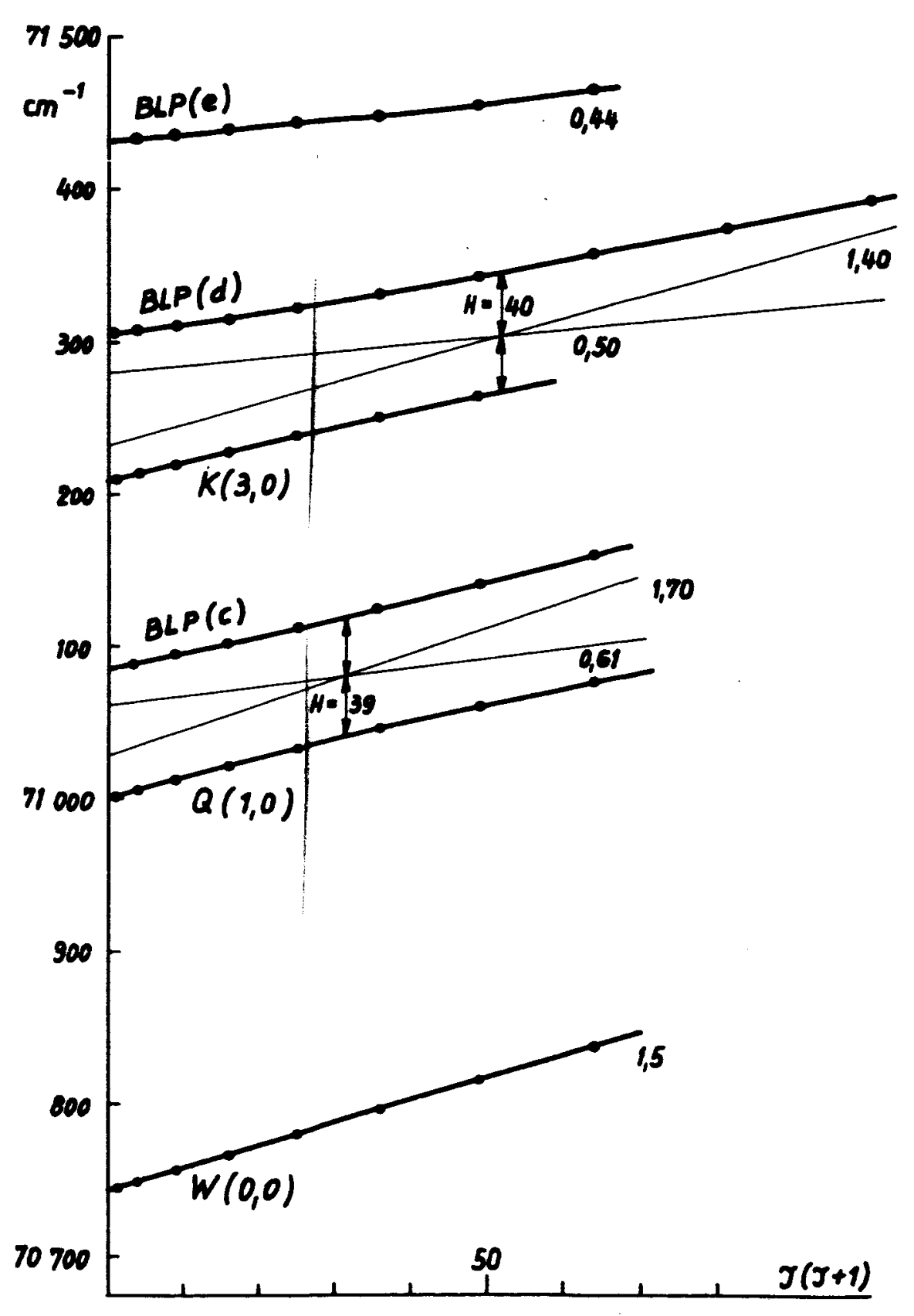



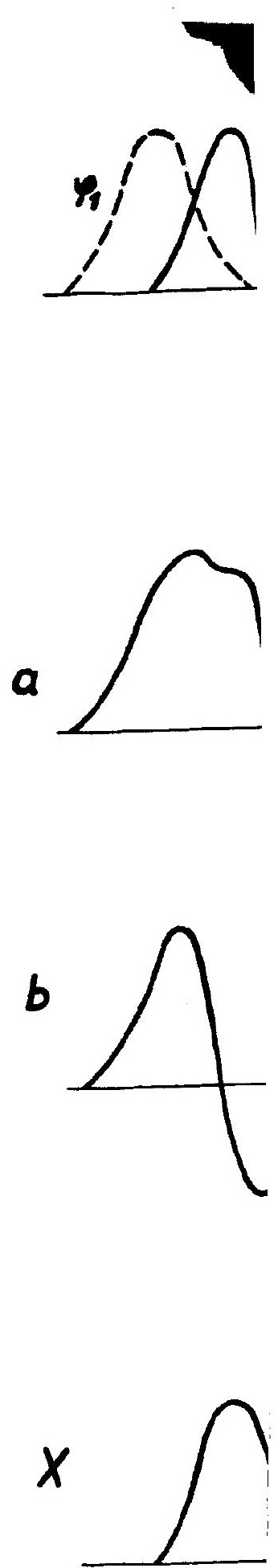

$:$ 


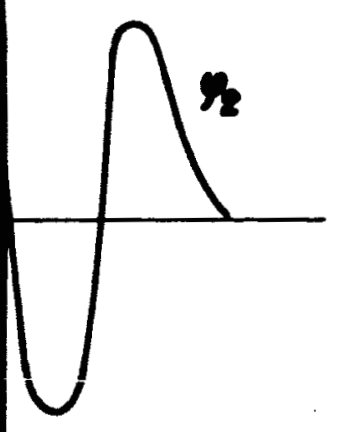

$\left.\begin{array}{l}\varphi_{1} \\ \varphi_{2}\end{array}\right\}$ unperturbed

$\varphi_{a}=\frac{1}{\sqrt{2}}\left(\varphi_{1}+\varphi_{2}\right)$

perturbed

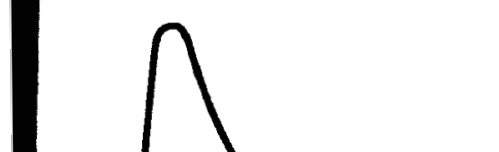

